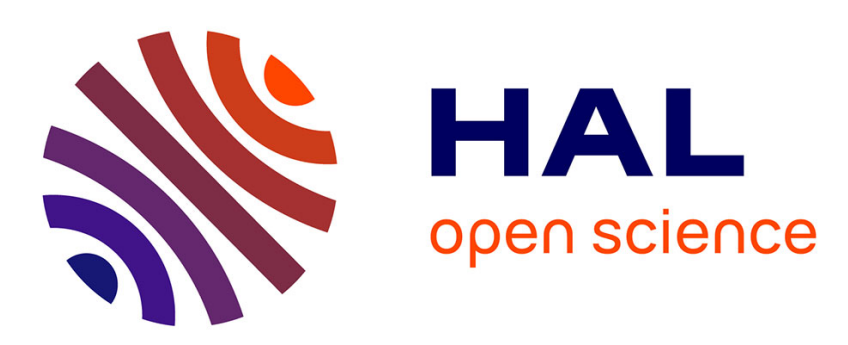

\title{
Participation of electric vehicle fleets in local flexibility tenders: Analyzing barriers to entry and workable solutions
}

Felipe Gonzalez, Marc Petit, Yannick Perez

\section{- To cite this version:}

Felipe Gonzalez, Marc Petit, Yannick Perez. Participation of electric vehicle fleets in local flexibility tenders: Analyzing barriers to entry and workable solutions. [Research Report] EUI-FSR. 2021. hal03420072

\section{HAL Id: hal-03420072 \\ https://hal.science/hal-03420072}

Submitted on 8 Nov 2021

HAL is a multi-disciplinary open access archive for the deposit and dissemination of scientific research documents, whether they are published or not. The documents may come from teaching and research institutions in France or abroad, or from public or private research centers.
L'archive ouverte pluridisciplinaire HAL, est destinée au dépôt et à la diffusion de documents scientifiques de niveau recherche, publiés ou non, émanant des établissements d'enseignement et de recherche français ou étrangers, des laboratoires publics ou privés. 

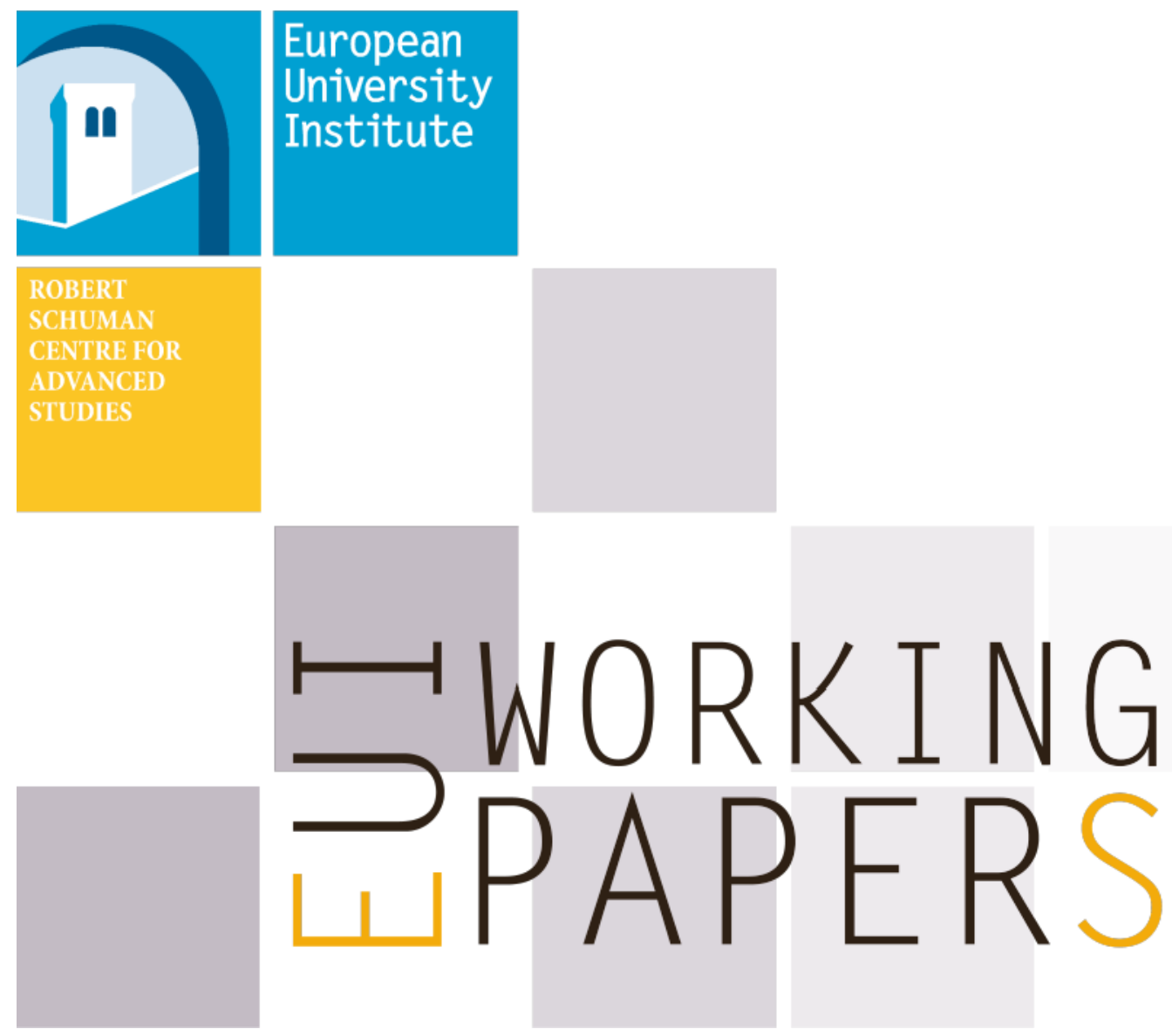

RSC 2021/12

Robert Schuman Centre for Advanced Studies Florence School of Regulation

Participation of electric vehicle fleets in local flexibility tenders:

Analyzing barriers to entry and workable solutions

Felipe Gonzalez Venegas, Marc Petit, Yannick Perez 

European University Institute

Robert Schuman Centre for Advanced Studies

Florence School of Regulation

Participation of electric vehicle fleets in local flexibility tenders: Analyzing barriers to entry and workable solutions

Felipe Gonzalez Venegas, Marc Petit, Yannick Perez

EUI Working Paper RSC 2021/12 
Terms of access and reuse for this work are governed by the Creative Commons Attribution 4.0 (CCBY 4.0) International license. If cited or quoted, reference should be made to the full name of the author(s), editor(s), the title, the working paper series and number, the year and the publisher.

ISSN 1028-3625

(C) Felipe Gonzalez Venegas, Marc Petit, Yannick Perez, 2021

This work is licensed under a Creative Commons Attribution 4.0 (CC-BY 4.0) International license. https://creativecommons.org/licenses/by/4.0/

Published in February 2021 by the European University Institute.

Badia Fiesolana, via dei Roccettini 9

I - 50014 San Domenico di Fiesole (FI)

Italy

Views expressed in this publication reflect the opinion of individual author(s) and not those of the European University Institute.

This publication is available in Open Access in Cadmus, the EUI Research Repository:

https://cadmus.eui.eu 


\section{Robert Schuman Centre for Advanced Studies}

The Robert Schuman Centre for Advanced Studies, created in 1992 and currently directed by Professor Brigid Laffan, aims to develop inter-disciplinary and comparative research on the major issues facing the process of European integration, European societies and Europe's place in $21^{\text {st }}$ century global politics.

The Centre is home to a large post-doctoral programme and hosts major research programmes, projects and data sets, in addition to a range of working groups and ad hoc initiatives. The research agenda is organised around a set of core themes and is continuously evolving, reflecting the changing agenda of European integration, the expanding membership of the European Union, developments in Europe's neighbourhood and the wider world.

For more information: http://eui.eu/rscas

The EUI and the RSC are not responsible for the opinion expressed by the author(s).

\section{Florence School of Regulation}

The Florence School of Regulation (FSR) is a partnership between the Robert Schuman Centre for Advanced Studies (RSC) at the European University Institute (EUI), the Council of the European Energy Regulators (CEER) and the Independent Regulators Group (IRG). Moreover, as part of the EUI, the FSR works closely with the European Commission.

The objectives of the FSR are to promote informed discussions on key policy issues, through workshops and seminars, to provide state-of-the-art training for practitioners (from European Commission, National Regulators and private companies), to produce analytical and empirical researches about regulated sectors, to network, and to exchange documents and ideas.

At present, its scope is focused on the regulation of Energy (electricity and gas markets), Communications \& Media, and Transport.

This series of working papers aims at disseminating the work of scholars and practitioners on current regulatory issues.

\section{For further information}

Florence School of Regulation - Communications and Media, Robert Schuman Centre for Advanced Studies European University Institute Villa Raimondi, 121/111 Via Boccaccio, I-50133, Florence, Italy

email: FSR.ComsMedia@eui.eu

website: https://fsr.eui.eu/communications-media/

phone: +39055.4685803 



\begin{abstract}
Distribution system operators (DSO) are starting to implement market-based mechanisms to use the flexibility offered by distributed energy resources (DER) such as electric vehicles (EV). Several European countries are trialing a range of real-life tests and market designs, and local flexibility tenders that allow DSOs to procure medium- to long-term flexibility have found early success. Here we set out to: i) identify the remaining barriers to entry for DER aggregators in these new flexibility schemes, and ii) quantify the participation of EV fleets in long-term flexibility tenders. We built a model to evaluate the potential EV aggregator gains on local flexibility tenders considering market rules, definitions of flexibility product, and different EV fleet compositions. Our model shows that the main parameters affecting EV fleet aggregator participation and remuneration are bidirectional capability (V2G), fleet reliability, and the right match-up between availability profiles and tender requirements. In best-case scenarios, EV fleet aggregators can expect revenues of over $€ 1400 / \mathrm{EV} /$ year providing services for only a few hours or months per year. The paper concludes with policy recommendations based on best practices to boost DER participation in local flexibility markets.
\end{abstract}

\title{
Highlights
}

- First long-term local flexibility tenders show potential high value of flexibility.

- Market rule analysis of five major European flexibility market schemes.

- Developed a model to quantify participation of DER in flexibility tenders.

- EV case study to identify main parameters affecting participation.

- Policy recommendations based on best practices to boost DER participation in local flexibility markets.

\section{Keywords}

Electric vehicles, Distribution systems, Smart grids, Flexibility markets, V2G, Smart charging 



\section{Introduction*}

Decarbonization and digitization are disrupting the way distribution grids are operated and planned. Additional load from cross-sector electrification, such as from electric vehicles (EVs) or heat pumps, and mass integration of distributed generation (solar panels and wind turbines) could create congest the grid congestion, requiring distribution system operators

(DSO) to make heavy infrastructure investments to maintain grid security and reliability. However, broad uptake of connected and controllable distributed energy resources (DER) like EVs or battery energy storage systems (BESS) offers ways to add flexibility to the system by adapting consumption and injection patterns, helping grid operators to defer or avoid reinforcements, or improving grid reliability and security of supply (see Thompson and Perez (2019) for a review of options).

DSOs have historically taken a 'fit-and-forget' approach to grid management by investing in oversized infrastructure to cope with additional peak demand or generation Knezovi'c et al. (2015). However, by adopting an active management approach to grid operation and planning, DSOs can procure flexibility from controllable DERs to make more efficient use of grid assets. Several use-cases have been identified. In the medium-to-long-term, flexibility could reduce or avoid grid reinforcements or reduce the risk of stranded assets under uncertain load growth. In the operational timeframe, flexibility can be used to solve grid congestion, improve grid reliability during maintenance periods, and provide back-up power in fault events (Knezovi'c et al. (2017); Gonzalez Venegas et al. (2019a)).

There are four main frameworks enabling DSOs to procure flexibility: grid codes, smart connection agreements, advanced network tariffs, and market-based solutions. Regulators encourage the marketbased option to ensure flexibility is procured in a competitive manner

(Council of European Energy Regulators (2018)).In this context, DSOs in the UK, France, Germany and the Netherlands have already started to implement market solutions to procure flexibility for the short- and long-term. Likewise, New York State utilities implement 'non-wires alternatives' where DERs can provide a cost-effective alternative to costly reinforcement investments (REV Connect (2020)).

EV sales in Europe are growing fast, increasing by $32 \%$ in 2018 and 50\% in 2019. In 2019, over 50,000 EV were sold in France, the UK and Netherlands, and over 100,000 in Germany. These countries have strong EV adoption objectives, with the Netherlands planning to ban the sale of new internal combustion vehicles by 2030 and the UK and France by 2040 (International Energy Agency (2020)).

Mass EV integration can pose challenges for grid operation and planning due to increased load, but it can also provide flexibility through smart charging (V1G) and vehicle-to-grid (V2G) (Gonzalez Venegas et al. (2019b), where the EV can be used as a mobile storage system that can provide power back to the grid. This has been proven technically for complex flexibility services like frequency regulation (Codani (2016), Codani et al. (2016)), with commercial applications currently running in the

\footnotetext{
Acknowledgement

This research benefits from the support of the Chair "Groupe PSA: Hybrid Technologies and Economy of Electromobility", so-called Armand Peugeot Chair, led by CentraleSupélec and the ESSEC and sponsored by Groupe PSA, and the financial support of the ANRT for the CIFRE contract n²018/0031.

The authors also thank Fabio Antonialli and Jean Hassler for their insightful comments that helped improve this work.

\section{CRediT authorship contribution statement}

Felipe Gonzalez Venegas: Conceptualization, Data Curation, Methodology, Investigation, Software, Writing - Original Draft, Writing - Review \& Editing, Visualization. Marc Petit: Conceptualization, Writing - Review \& Editing, Supervision, Funding Acquisition. Yannick Perez: Conceptualization, Writing - Review \& Editing, Supervision, Funding acquisition.
} 
US, France and Denmark (Banol Arias et al. (2018), Nuvve Corp (2017)). EVs therefore position as one of the main candidates to participate in emerging distribution flexibility markets (Gonzalez Venegas et al. (2020a)).

The objective of this work is to quantify the revenues EV fleets can obtain by participating in emerging local flexibility markets and the impact of the governing tender rules, in line with the stream of research developed in Borne et al. (2018b,a). With that vision, we analyzed the rules of emerging flexibility markets to identify the main barriers to participation of EVs. We go on to quantify the participation of three types of EV fleets in medium- to long-term flexibility tenders by implementing a Monte Carlo simulation-based methodology that captures the stochasticity of EV usage profiles.

This work makes four main contributions.. First, we analyzed the impact of local flexibility tender rules on the participation of DERs. To the best of our knowledge, no studies have tackled long-term market mechanisms at the distribution level. Second, we proposed a two-stage methodology for evaluating EV fleet participation in long-term tenders that can capture various specificities of the tender process. Third, we quantified EV fleet participation using real-world data from demonstrator projects in Europe, which makes the results representative. And fourth, we formulate policy recommendations based on best-practices to boost the participation of DERs in local flexibility markets.

The paper is structured as follows. Section 2 outlines the emerging flexibility markets in Europe and identifies key market rules that can affect EV participation. Section 3 explains the methodology used to quantify EV participation in long-term distribution tenders and presents the case study. Section 4 reports the main results of the study on EV remuneration.

Section 5 completes the work with some key conclusions and policy recommendations.

\section{Looking for decentralized flexibility markets}

In recent years, the use of flexibility for distribution systems has gained a lot of attention from the scientific community, industry (see EDSO et al. (2018) and Cedec et al. (2019)) and regulators (Council of European Energy Regulators (2018)). Indeed, mass integration of DERs will require an active management of the distribution grid by making use of controllable assets to improve grid operation and planning.

Academic studies have mostly focused on local flexibility trading platforms that allow DSOs to solve congestion in (near-) real time (see Olivella-Rosell et al. (2018) and Fonteijn et al. (2020) ) for examples of implementations in the Invade and Interflex demonstrator projects, and Jin et al. (2020) for a review of methods and models). However, DSOs looking to procure flexibility to manage investment deferral need to have a long-term perspective, as they face high risks if they rely solely on short-term markets. Local flexibility markets may suffer from a lack of liquidity due to their limited size, thus exposing DSOs to high flexibility prices or even endangering grid reliability since DSOs would have limited options if the market fails. A classical solution to this problem is long-term contracting as defined by Williamson (1985) and Williamson (1996). Long-term contracts are an appropriate way to manage risks between participants. In our case, they provide a degree of certainty to both contractors: DSOs are guaranteed that flexibility will be available if needed ${ }^{1}$, and flexibility operators are provided with secured future revenue streams.

As discussed in Schittekatte and Meeus (2020), long-term contracts also can mitigate gaming in short-term markets. Gaming can be serious issue in local flexibility markets where the limited number of market participants can reduce competition and where a participant with sufficient knowledge of expected grid bottlenecks can aggravate congestion and then get paid to solve the problems created by itself.

1 Or reducing the risk of stranded assets in case the expected load growth does not materialize. 


\subsection{Emerging flexibility markets}

We have identified five European initiatives surrounding the implementation of local flexibility markets, each taking a different approach according to their local requirements. Two of them, UKPN and Enedis (DSOs), propose mid- to long-term tenders to procure flexibility ahead of time, as their major use-case is to defer grid reinforcement. The other three, Enera, Nodes and GOPACS, are short-term (intraday) market platforms whose main use-case is to reduce renewable energy source (RES)-driven congestion at transmission or distribution level. Schittekatte and Meeus (2020) analyzed four of these projects through the lens of controversies around local flexibility market design, including level of integration of local markets into existing structures (day-ahead, intraday, and balancing markets), roles and responsibilities of DSOs in market operation, standardization of flexibility products, and level of coordination between DSO and transmission system operator (TSO).

First, UK Power Networks (UKPN, the London-area DSO) has implemented local flexibility tenders since 2018 to contract flexibility for the medium- to long-term in sections of the grid where they expect congestion, allowing them to reduce investment costs (UK Power Networks (2018)). UKPN identifies periods of time during which they expect congestion, usually winter evening hours due to peak load, and the amount of flexibility required to solve them. Flexibility is required to be available at these periods ('availability windows') but not necessarily activated each day, as activations are expected to happen only a few hours per year.

The April 2020 tender process comprised 62 high voltage (HV) zones and over 60 low voltage (LV) zones, awarding contracts for $42 \mathrm{HV}$ and $15 \mathrm{LV}$ zones, for up to 7 years duration. EV companies won a significant share of the flexibility contracts, for a total $36 \mathrm{MW}$ out of $52 \mathrm{MW}$ awarded in the HV tender (UK Power Networks (2020)). HV tenders were a competitive process, where participants bid an availability fee (in $\mathrm{f} / \mathrm{MW} / \mathrm{h}$ ) and a utilization fee (in $\mathrm{f} / \mathrm{MWh}$ ) for a given amount of flexibility. Results for the April 2020 tender (Fig. 1) show that prices vary widely depending on participant strategy and tender conditions. Availability payments (left plot) can go from $£ 3$ to over $£ 4500 / \mathrm{MW} / \mathrm{h}$, which is significantly higher than payments for frequency regulation services in continental Europe ${ }^{2}$ and the $\mathrm{UK}^{3}$ which are under $€ 10$ or $€ 10 / \mathrm{MW} / \mathrm{h}$. Equivalent payments per firm $\mathrm{kW}$ (right plot) ${ }^{4}$ average $£ 61.3 / \mathrm{kW} /$ year but can exceed $£ 300 / \mathrm{kW} /$ year in certain areas, showing the potential high value of flexibility for investment deferral. To simplify flexibility procurement at LV level, the LV tender provided only a fixed service fee of $£ 47.58 / \mathrm{kW} /$ year, which is close to the average payments for their HV tender.

Additionally, contracts were signed with flexibility providers to deal with other grid requirements, such as managing outages. Flexibility activation occurs in real-time according to grid conditions, but the flexibility providers have no obligation to provide the flexibility.

These contracts therefore only entail utilization payments.

2 Payments for frequency containment reserve (FCR) in the FCR Cooperation averaged $€ 7.4 / \mathrm{MW} / \mathrm{h}$ between January 2017 and August 2020 (RTE Portail Service (2020)). FCR Cooperation is a common reserve market for FCR between TSOs in France, Germany, Switzerland, Belgium, the Netherlands and Austria

3 Most of the firm frequency response services contracted by NGESO (UK's TSO) are between f0-4/MW/h National Grid ESO (2020).

4 Service payments per firm $\mathrm{kW}$ are computed for each accepted bid considering the number of hours during which flexibility should be available and a total of 10 one-hour activations per year. Serv.Payment $[\mathrm{f} / \mathrm{kW}]=$ Act.Payment $[\mathrm{f} / \mathrm{kWh}] * 1[\mathrm{~h}] * 10$ + Avail.Payment[f/kW.h]*Avail.Hours[h] 


\section{Figure 1: Results for accepted bids during the UKPN April $2020 \mathrm{HV}$ tender. Availability and utilization prices (left) and equivalent service payments per firm $\mathrm{kW}$ (right). Marker sizes are proportional to bid flexibility $[\mathrm{kW}]$}
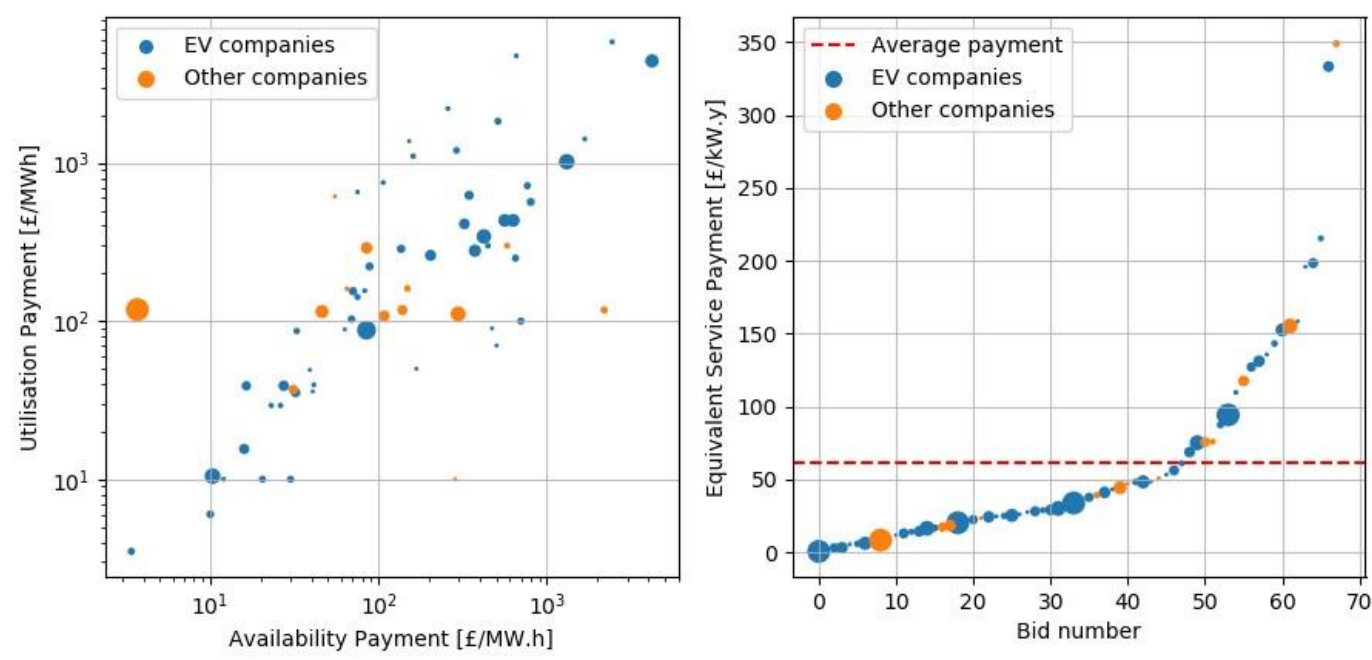

Second, Enedis, the French DSO supplying 95\% of continental France, launched its first tender process during 2019-2020. The tender covers six zones in their medium voltage (MV) grid, each with different use-cases. Three zones require flexibility for investment deferral, with availability and utilization payments in much the same way as the UKPN HV tenders, and three zones require flexibility on the operational timeframe, with only utilization payments. The use-cases for investment deferral are also varied, with one case dealing with peak load, a second one dealing with voltage regulation due to high PV penetration and requiring reactive power, and a third one to guarantee reliability under fault conditions, thus requiring flexibility to be available at all times (Enedis (2020a)). Results for the first tender process are expected by end-2020.

Third, GOPACS is a collaboration between Dutch grids operators, TenneT, the TSO, and four DSOs. It provides a platform that serves as an intermediary between grid operators and the intraday market platform ETPA, operational in the Netherlands. Participants of the ETPA trading platform can respond to GOPACS requests by submitting bids with a locational tag (Schittekatte and Meeus (2020)).It has so far only been used to solve transmission-level congestion (GOPACS.eu (2020)), as an alternative to redispatching, but they expect to use it for distribution-grid needs in the near future.

Fourth, Enera is a German pilot project allowing DSOs EWE NETZ and Avacon and TSO TenneT to reduce uneconomic curtailment of excess RES generation. It proposes intraday trading based on the existing market platform Epex Spot (EPEX Spot (2018)). Likewise, NODES is running two pilot projects in Germany in an effort to reduce curtailment in wind-saturated regions by providing an intraday marketplace (Engelbrecht (2019)). Both of these projects thus require load increase to absorb extra renewable generation.

These projects illustrate how solutions are tied to problems faced by DSOs. Enera and NODES face a problem of excessive RES generation where the current solution is uneconomic curtailment, so they proposed a short-term platform to improve grid operation. UKPN and Enedis mainly face issues that require grid reinforcements, and so they implemented tender processes to ensure flexibility availability and improve grid planning. Physically, flexibility needs are related to load reduction or load increase 
(active power) or reactive power exchanges. For these local needs, the flexible resources must be well located to bring an efficient solution.

\subsection{Can DERs participate in emerging flexibility markets?}

A modular framework has been developed to identify barriers to entry for DERs in local flexibility tenders (see Gonzalez Venegas et al. (2019a)). This modular framework serves to classify market rules and identify best practices and room for improvement. Here we extend this initial frame to include penalties not considered in the previous analysis. We apply this extended framework to emerging flexibility markets.

Our modular framework is comprised of three hierarchical modules ${ }^{5}$, as summarized in Table 1. Here we adapt this framework by including parameter C.4, Penalties, which are the penalties incurred by a flexibility operator if it does not respond to full extent to a flexibility request by the DSO. While the DSO's need for security is understandable given the limited availability of local flexibility resources, by implementing penalties, they transfer this issue to flexibility operators. Therefore, EV or demand response (DR) aggregators have to contend at the same time with high uncertainty on their flexibility availability profiles and high penalties. High penalties can thus prove a barrier to entry for these actors.

Table 1: Modular framework to evaluate the participation of DERs in local flexibility markets

\begin{tabular}{lll}
\hline Module & \multicolumn{2}{l}{ Parameter } \\
\hline Administrative rules & A1 & Technical discrimination \\
& A2 & Flexibility platform interoperability \\
Product definition & B1 & $\begin{array}{l}\text { Distance from real-time and availability } \\
\text { window definition }\end{array}$ \\
& B2 & Minimum activation time \\
& B3 & Minimum bid size \\
& B4 & Location \\
Payment scheme & C1 & Nature of payment \\
& C2 & Baseline definition \\
& C3 & Stacking of services \\
& C4 & Penalties \\
\hline
\end{tabular}

We assess the performance of emerging local flexibility tenders using the modular framework shown in Table 2. This table shows best practices among the different market designs and allows to identify differences in implementation.

The main barriers appear on lack of properly defined connection and metering requirements for DERs (Everoze and EVConsult (2018), Commission de Régulation de l'Energie (2018)), high minimum bid requirements (above $500 \mathrm{~kW}$ for participants) for most implementations, and poorly defined baseline

5 See Gonzalez Venegas et al. (2019a) for a full description of the framework. 
definitions. Previous work by Gonzalez Venegas et al. (2020b) analyzed the impact of minimum bid sizes and baseline definitions on the participation of EV fleets. Setting a minimum bid size of $500 \mathrm{~kW}$ can require the aggregation of 60 to $200 \mathrm{~V} 2 \mathrm{G}$-capable EVs, which may prove extremely hard to accomplish in restricted tender areas, and an even higher number if we only consider only unidirectional charging (V1G). Furthermore, poorly defined baselines ${ }^{6}$ may not reflect real flexibility activation, as they can under-reward or over-reward flexibility. Setting well-adapted baselines is a challenging task, but it has already been addressed for flexibility services for transmission systems, such as explicit demand response, as discussed by Rossetto (2018).

Implementations differ on several aspects. On platform interoperability and access, Piclo Flex (the platform used by UKPN), Enedis and GOPACS have the support of most DSOs in their countries, and Enera can employ the Epex Spot platform widely used in Europe, but NODES is a new entrant with only pilot implementations. Trading platforms like Epex Spot (Enera) and ETPA (GOPACS) have utilization fees that could potentially be barriers to new entrants. Most differences arise from the grid issues they tackle. UKPN's tender deals mostly with load-related grid reinforcements, allowing for welldefined availability windows during expected peak-demand periods, whereas the Enedis tender tackles different investment deferral problems, and thus has a diverse array of availability windows and flexibility requirements. On the other hand, short-term market platforms do not need availability windows, and their technical characteristics (minimum bid size, duration of service, baselines, utilization payments only) are aligned with energy market platforms, which can ease the participation for aggregators already active in these platforms. For example, GOPACS trades 15-minute blocks of energy through the ETPA platform, and baselines are dependent on the aggregators' t-prognosis ${ }^{7}$.

The conditions governing penalties also differ in terms of level and application. UKPN's implementation has a light penalty scheme, with only a reduction of payment for under-delivered flexibility as a way to facilitate the entry for new market participants. UKPN can exclude a participant if they fail to deliver flexibility more than three times (UK Power Networks (2020)). Enedis has stated that it intends to align its penalties to the TSO balancing mechanism and retain the possibility of banning market participants after repeated default events (Enedis (2020b))Short-term platforms do not yet feature penalties but expect to introduce them in the future Schittekatte and Meeus (2020).

The UKPN tender process is the most mature flexibility market solution, with sustained growth since its beginnings and a highly transparent tender process $^{8}$. It has shown that flexibility for investment deferral can have great value, with strong participation by EV aggregators as flexibility providers. Therefore, for the rest of this work, we aim to quantify the potential participation of EV fleets in longterm flexibility tenders. The goal is to identify key parameters that can impact remuneration. The parameters to be analyzed are availability window definition, duration of service (as flexibility requirements can run up to 3 hours in the case of a sustained congestion event), and the conditions surrounding penalties.

\section{Methodology and case study}

This section presents the model developed to quantify the economics of EV fleet participation in distribution flexibility tenders and the associated case study.

6 Baselines are the counterfactual load or generation level of the flexible assets if they had not provided flexibility. The flexibility delivered to the system is measured against this baseline.

7 t-prognosis are the self-declared expected profile of the aggregator

8 Before the tender, UKPN signals the expected revenue range per tendered zone, informing market participants of the expected value of flexibility at each zone, and after the tender they publish the bids filed under the tender process. Pre- and post-tender reports are available at https://smartgrid.ukpowernetworks.co.uk/flexibilityhub/ 
Table 2: Assessment of emerging flexibility markets rules

\begin{tabular}{|c|c|c|c|c|c|c|c|}
\hline & Rule & Ideal & UKPN & Enedis & GOPACS & Enera & NODES \\
\hline A1 & Technical discrimination & $\begin{array}{l}\text { Adapted connection \& } \\
\text { metering requirements }\end{array}$ & +- & +- & +- & N.I. & N.I \\
\hline A2 & $\begin{array}{l}\text { Flexibility platform } \\
\text { interoperability }\end{array}$ & Ample support, open access & + & + & +- & +- & - \\
\hline B1.1 & Distance to activation & According to DSO's need & Long & Long & + & + & + \\
\hline B1.2 & Availability window definition ${ }^{a}$ & Well defined/shorter & + & +- & N.A & N.A. & N.A. \\
\hline B2 & Minimum duration of service & $\begin{array}{l}\text { No greater than energy } \\
\text { trading period }(15-30 \mathrm{~min})\end{array}$ & + & + & ++ & + & + \\
\hline B3 & Minimum bid size & Low $(<=50 \mathrm{~kW})$ & $+/++$ & - & - & N.I. & N.I. \\
\hline B4 & Location & Well defined & + & +- & + & N.I. & +- \\
\hline $\mathrm{C} 1$ & Nature of payments & Adapted to product & $+(A v+U t)$ & $+(A v+U t)$ & $+(U t)$ & $+(U t)$ & $+(U t)$ \\
\hline $\mathrm{C} 2$ & Baseline definition & Adapted to technology & +- & + & + & + & + \\
\hline C3 & Stacking of services & Possible & + & + & N.A. & N.A. & N.A. \\
\hline C4 & Penalties & Low/Proportionate & + & +- & + & + & + \\
\hline
\end{tabular}

${ }^{\mathrm{a}}$ A clearly-defined availability window is only required for long-term tenders.

+ /- indicate positive/negative assessment of implementation.

N.A.: Not Applicable, N.I.: No information available.

Ut: Utilization payment. Av: Availability payment

\subsection{Method and modelling}

We developed a methodology that emulates the flexibility tender process. In a first stage, we evaluate the potential participation of EV fleets in medium- to long-term flexibility tenders, and in the second stage, we evaluate how these fleets perform in terms of effectively delivering the committed flexibility to grid operators, and compute the allied payments and penalties. Each stage has two sub-modules, as summarized in Fig. 2.

\section{Figure 2: Two-stage methodology to quantify EV participation in flexibility tenders}

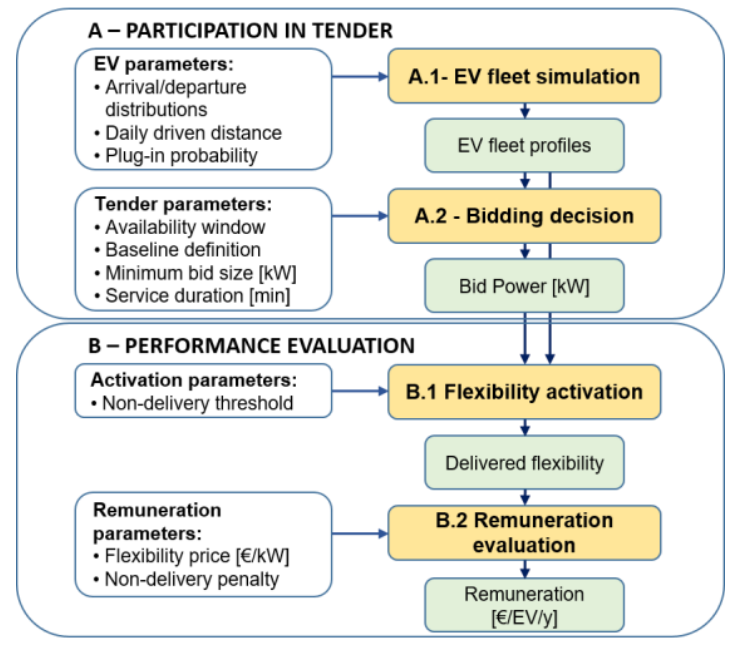

\subsubsection{Stage A: Participation in tender}

This stage emulates the bidding decision process to participate in the tender. EV aggregators participating in flexibility tenders commit to delivery flexibility months (or even years) in advance. Flexibility availability depends on EV-user patterns, which can be highly uncertain, as V2G-based 
flexibility can be only assured when the vehicle is plugged in. EV aggregators will need to forecast EV usage patterns (sub-module A.1) and then decide on the amount of flexibility they will bid on the tender (sub-module A.2).

A.1 Monte Carlo simulations of EV fleet patterns. Here we compute EV charging and flexibility profiles for multiple EV fleets using a multi-agent-based simulation described in Gonzalez Venegas et al. (2019c). Each EV has a set of stochastic parameters: daily travel distance (fixed for each EV) and arrival and departure times (variable each day). We consider a probabilistic non-systematic plug-in behavior from EV users in which users do not plug in their vehicle every day or can be absent from their usual charging point, as described Gonzalez Venegas et al. (2020b). This emulates the behavior observed in real-life conditions, as in Western Power Distribution (2019).

A.2 Bidding decision. Given the expected EV charging and flexibility profiles and tender rules, we compute the EV fleet bid power (in $\mathrm{kW}$ ). To achieve this, first we compute the baseline upon which flexibility will be measured from the simulated EV charging profiles, then we evaluate the flexibility that can be provided to the system with respect to the baseline, and determine the bid as the flexibility that can be provided at a given confidence level (in this case $90 \%$ ).

\subsubsection{Stage B: Performance evaluation}

Tender participants will be evaluated on their ability to deliver the committed flexibility when required. In case of under-delivery, they can face high penalties and even be banned from the market. We simulate flexibility activation events (sub-module B.1) and then compute payments and penalties (sub-module B.2). Penalties depend on two parameters: the penalty threshold, where delivery of flexibility under this threshold triggers penalties, and the penalty value, in percentage of expected payment.

B.1 Flexibility activation. We simulate activation events by randomly sampling flexibility events during the availability windows and evaluating flexibility delivery with respect to the penalty threshold.

B.2 Remuneration evaluation. We compute payments and penalties. If the fleet is unable to deliver full amount of the committed flexibility but is still considered a successful flexibility activation (flexibility delivery is above the penalty threshold), then payments are reduced accordingly. If the delivered flexibility is below the penalty threshold, then penalties are applied in addition to the reduction of payment. This emulates the UKPN de-rating performance factor and the Enedis penalty implementation scheme.

\subsection{Case studies}

We evaluate tender participation for three types of fleets doing uncontrolled overnight charging: one company fleet with consistent travel schedules, and two commuter fleets with highly variable travel patterns and non-systematic plug-in behavior (i.e. not plugging in every day). The three fleets are comprised of $30 \mathrm{EVs}$ in line with the minimum fleet size requirements found in Gonzalez Venegas et al. (2020b), ), can provide unidirectional (V1G) and bidirectional (V2G) flexibility with a $7 \mathrm{~kW}$ charger that has $95 \%$ charging and discharging efficiency, and the EVs have a $50 \mathrm{kWh}$ battery pack in line with current battery size trends.

The EVs are modeled with stochastic parameters on daily travelled distances and arrival and departure times. Statistical distributions for the company fleet are based on data from the Parker project, shown in Table 3, and the EVs are always plugged in after the last trip of the day (Berthou (2018); Andersen et al. (2018)). The commuter fleets are modeled based on data from the Electric Nation project (dataset in Western Power Distribution). Arrival and departure patterns are modelled through a bivariate distribution, shown in Fig. 3, and daily travelled distances follow a log-normal distribution with a mean daily distance of $40.3 \mathrm{~km}$. One commuter fleet has low plug-in probability (Commuter LP), in line with 
Electric Nation data with an average plug-in ratio of 2.75 charging sessions per week, while the second commuter fleet (Commuter HP) has a higher plug-in ratio averaging 5.32 charging sessions per week, emulating the case where the aggregator incentivizes end-users to plug in. We carry out simulations for 50 weeks and 1000 fleets to capture the variability in end-user behavior.

Table 3: Stochastic parameters of studied fleets

\begin{tabular}{llccc}
\hline Parameter & Type & $\mu$ & $\sigma$ & Shift \\
\hline Company Fleet & & & & \\
Arrival time [h] & Normal & 13.2 & 1.82 & - \\
Departure time [h] & Log-normal & 1.04 & 0.53 & 5.5 \\
Daily distance $[\mathrm{km}]$ & Log-normal & 2.36 & 0.79 & - \\
Commuter Fleets & & & & \\
Daily distance $[\mathrm{km}]$ & Log-normal & 3.43 & 0.73 & - \\
\hline
\end{tabular}

\section{Figure 3: Joint probability distribution (left) and marginal distribution (right) for arrival and} departure times for commuter fleets
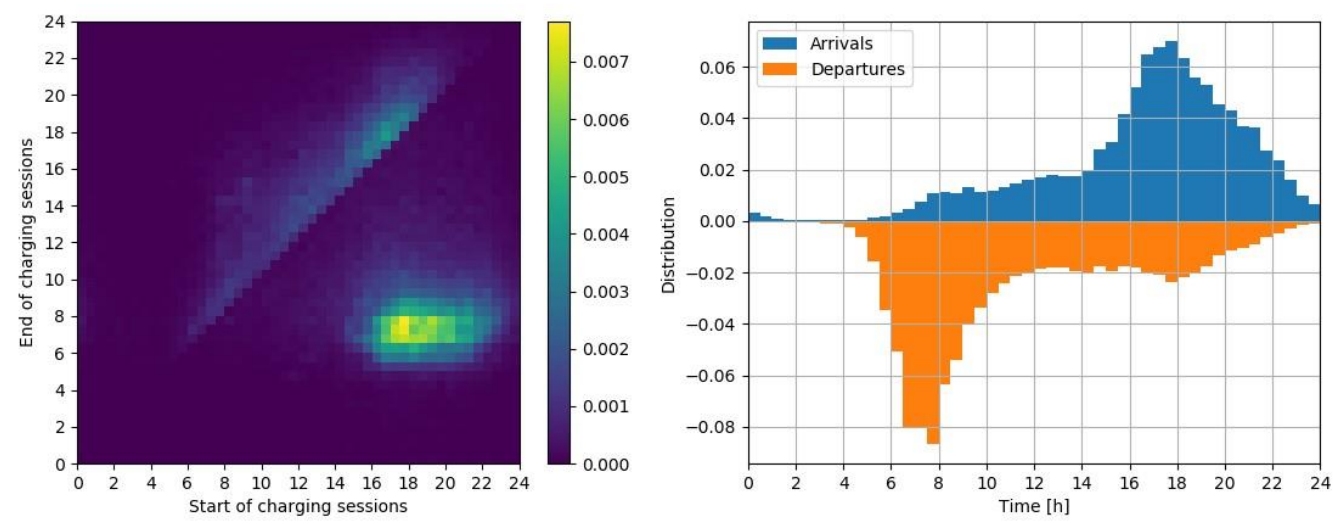

Regarding tender parameters, we considered two availability windows: an evening window from $5 \mathrm{pm}$ to $8 \mathrm{pm}$, consistent with a majority of zones tendered by UKPN, and a full-day window, consistent with one of the Enedis cases. Flexibility should be available for three months, and only during weekdays (a total of 60 days per year). A 30-minute 'downwards flexibility' (reducing demand or increasing generation) is required during the availability windows, though not necessarily activated every day.

We then consider the single-value baseline used in UKPN tenders, computed as the average power (consumed or generated) during the availability window on representative historical days, thus providing a single value for the whole availability window. While this baseline does not correctly capture load profiles, thus under- or over-rewarding flexibility, it does provide a good trade-off between simplicity and accuracy, especially for short availability windows. Further discussion on this baseline can be found in Gonzalez Venegas et al. (2020b), and on general baseline methodologies in Rossetto (2018). To evaluate the performance of flexibility delivery (sub-module B.1), we simulated 10 activations during the availability period.

We considered a minimum bid size of $50 \mathrm{~kW}$ per fleet, again in line with UKPN tenders. A low minimum bid size is crucial to allow distributed resources to participate in local tenders and foster competition in the local market. As discussed in Gonzalez Venegas et al. (2020b), minimum bid sizes of $500 \mathrm{~kW}$, such as those required by Enedis or GOPACS, might require fleets of hundreds of EVs, which may not be possible to find in limited geographical zones or remote areas. 
As shown in Fig. 1 (Section 2), remuneration can vary greatly according to tender competition and requirements. We considered an equivalent service payment of $€ 50$ per firm $\mathrm{kW}$, similar to the fixed price proposed in UKPN $2020 \mathrm{LV}$ tenders and close to the average of bids accepted in the UKPN 2020 $\mathrm{HV}$ tender. This represents an availability price of $€ 277 / \mathrm{MW} / \mathrm{h}$ for the evening window ( 3 hours per day for 60 days $=180 \mathrm{~h} / \mathrm{y}$ ) and $€ 35 / \mathrm{MW} / \mathrm{h}$ for the full-day window ( 24 hours per day for 60 days $=1440$ $\mathrm{h} / \mathrm{y})$.

Finally, we considered the penalty parameters implemented by UKPN and Enedis ${ }^{9}$, and a high penalty scenario. Table 4 shows the thresholds for successful flexibility activation and the penalty values in percentage of the remuneration price. A penalty of $0 \%$ (UKPN case) means that in the event of failed activation, providers get no penalties but no payment either.

Table 4: Penalties parameters

\begin{tabular}{lcc}
\hline Scenario & Threshold & Penalty \\
\hline UKPN & $60 \%$ & $0 \%$ \\
Enedis & $80 \%$ & $35 \%$ \\
High & $90 \%$ & $70 \%$ \\
\hline
\end{tabular}

\section{Results}

\subsection{Availability profiles and baseline}

The three fleets under study present different charging profiles and flexibility potentials, as shown in Fig. 4. Charging sessions for company fleets start earlier, with peak charging around $3 \mathrm{pm}$, and by $5 \mathrm{pm}$ all EVs are usually plugged in, thus offering maximum V2G flexibility potential during the evening availability window. Charging patterns are more variable for commuter fleets, partially coinciding with the evening peak window. Arrivals are more spread out during the afternoon, with peak simultaneous connections occurring after $11 \mathrm{pm}$. Since EVs are not connected every day, the ability of commuter fleets to provide flexibility is greatly reduced in comparison to the company fleet, which can be seen in the reduced V2G flexibility potential curve. Flexibility profiles for commuter fleets also present higher variability, as the number of EVs connected vary each day. This can present a challenge for aggregators that want to make use of EV flexibility.

EVs are not connected all day long, which means there are periods of the day where almost no flexibility is available from the fleets (typically between $8 \mathrm{am}$ and $4 \mathrm{pm}$ ), which leaves aggregators participating in full-day window tenders exposed to high risk of flexibility being activated while the fleet is unable to respond.

9 Enedis aligned its penalties to RTE's (French TSO) balancing mechanism (mécanisme d'ajustement, RTE (2020)) 
Figure 4: Charging profiles and V2G potential for a 30-minute service, and examples of VxG flexibility levels. Fleet size: 30 EVs. Lines represent average profiles and shaded areas represent profiles at $95 \%$ confidence level

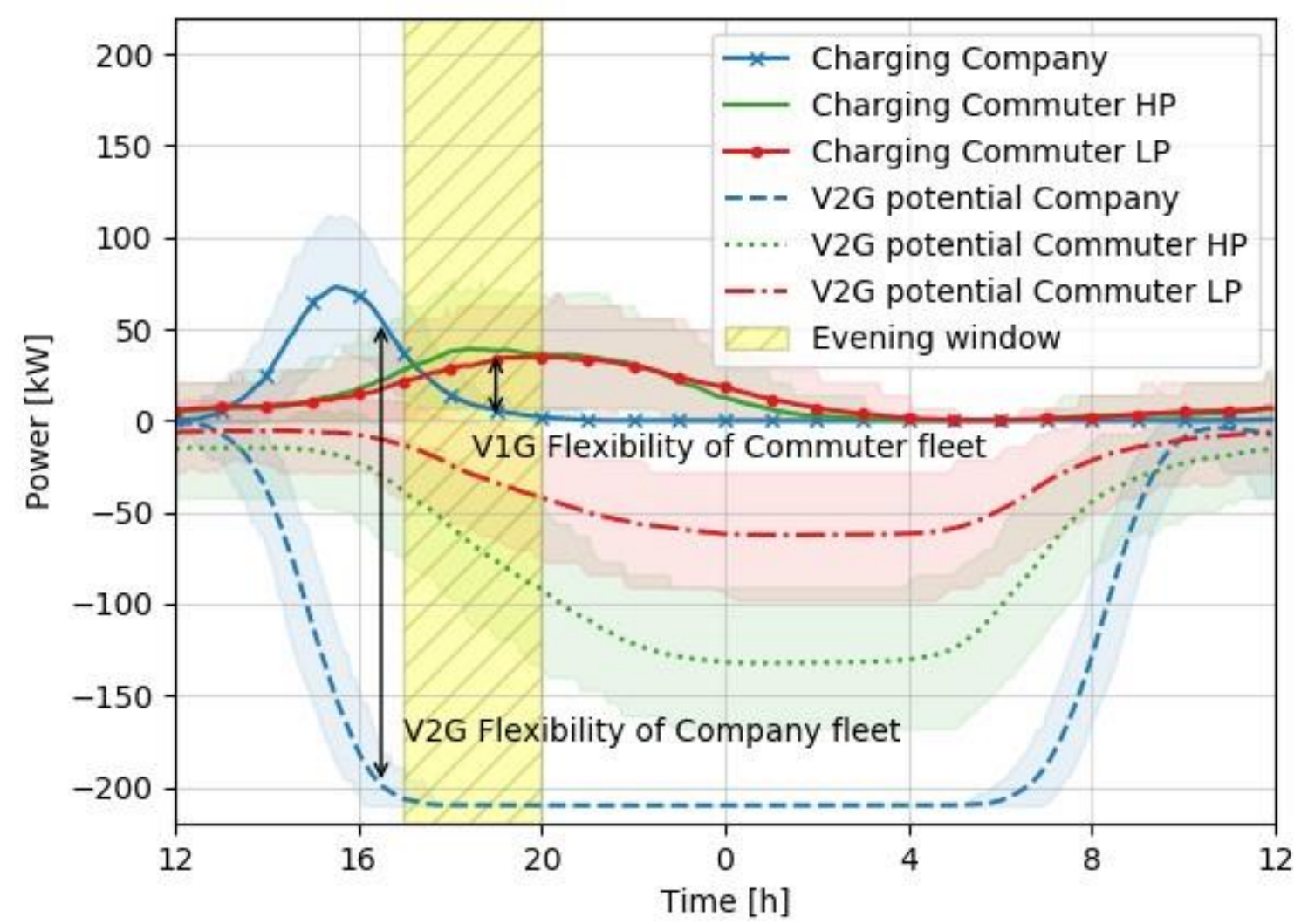

Baselines are computed for each fleet and each availability window as the average charging profile (the single-value baseline from UKPN). The model simulates a large number of fleets, each with different travel and charging patterns and therefore different baselines. Table 5 gives the average, minimum and maximum values for these baselines (in $\mathrm{kW} / \mathrm{EV}$ ) . Due to the coincidence of charging with the evening window, baselines for the commuter fleets are higher than for the company fleet, even though they only reach around $1 \mathrm{~kW} / \mathrm{EV}$. Thus, the recognized flexibility attainable by V1G-only solutions is therefore limited (maximum delivery of flexibility will mean completely stopping EV charging).

Table 5: Baseline average, minimum and maximum values [ $\mathrm{kW} / \mathrm{EV}]$ for the evening $(5 \mathrm{pm}-8 \mathrm{pm})$ and full-day availability windows. Fleet size: $30 \mathrm{EVs}$

\begin{tabular}{lcccccc}
\hline$[\mathrm{kW} / \mathrm{EV}]$ & \multicolumn{3}{c}{ Evening window } & \multicolumn{3}{c}{ Full-day window } \\
Fleet & Min & Avg. & Max & Min & Avg. & Max \\
\hline Company & 0.16 & $\mathbf{0 . 3 1}$ & 0.51 & 0.21 & $\mathbf{0 . 2 5}$ & 0.30 \\
Commuter HP & 0.85 & $\mathbf{1 . 0 0}$ & 1.16 & 0.31 & $\mathbf{0 . 3 6}$ & 0.41 \\
Commuter LP & 0.76 & $\mathbf{0 . 7 8}$ & 0.93 & 0.29 & $\mathbf{0 . 3 5}$ & 0.40 \\
\hline
\end{tabular}




\subsection{Minimum service duration}

We investigated the impact of minimum flexibility product service duration. Current tenders require a minimum service duration of 30 minutes, but also state that requirements can stretch up to 3 hours and that assets able to provide flexibility for longer periods will be privileged. Fig 5 shows the bid flexibility for the three fleets for service run times of 30, 60 and 120 minutes. For the evening window, bids for the three service durations present little difference (under $0.1 \mathrm{~kW} / \mathrm{EV}$ for the same type of fleet), meaning that EV fleets are capable of providing flexibility services for 120 minutes with the same reliability than for 30 minutes. This is because battery capacities are large enough to sustain a 120minute service (14 kWh represent only $28 \%$ battery capacity) and the flexibility window lies at the beginning of the fleet charging sessions, thus leaving ample time to recharge the battery ahead of nextday departure. The additional cycling induced by this flexibility service can increase battery degradation, but Wang et al. (2016) showed that providing 120-minute peak shaving services 20 times a year, similar to our case study, would reduce battery capacity by less than $0.5 \%$ over a 10 -year period, which is negligible compared to the $31 \%$ induced by driving and calendar aging.

\section{Figure 5: Bids [kW/EV] for 30-, 60- and 120-minute service durations. Fleet size: $30 \mathrm{EVs}$}

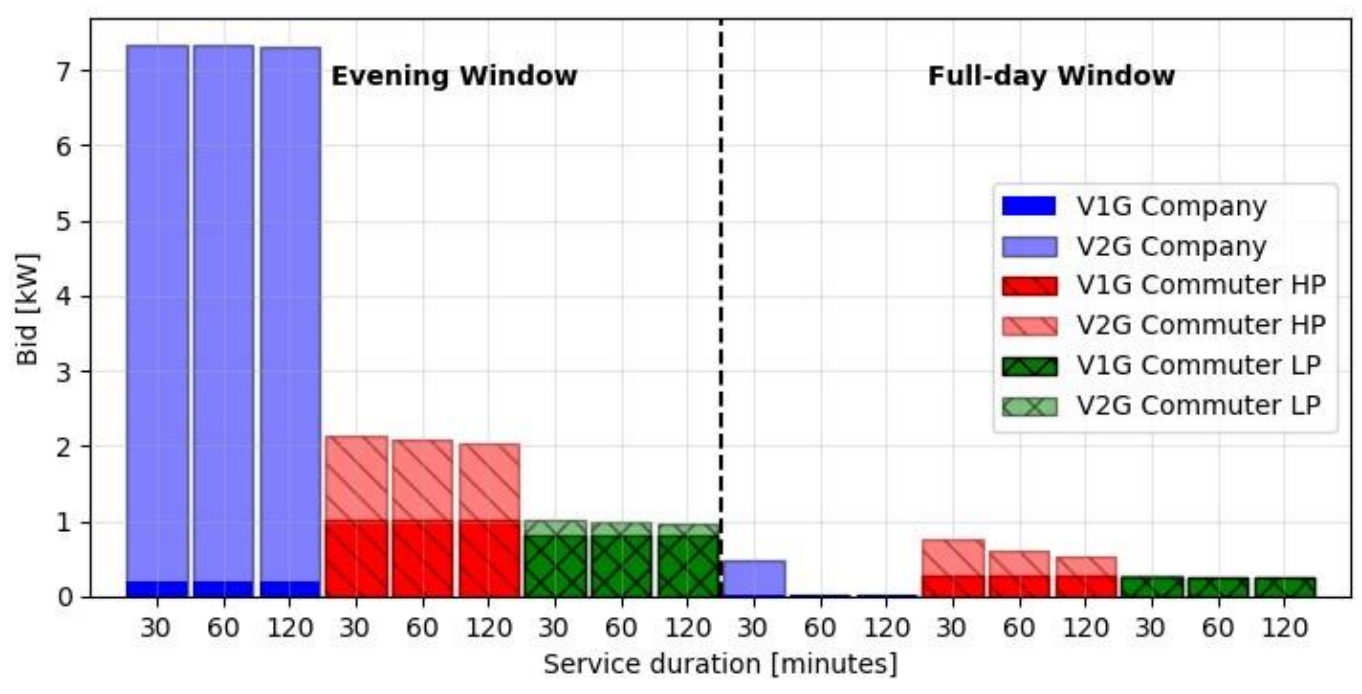

\subsection{Confidence level and penalties}

As shown in Fig. 4, there is high inter-day and intra-day variability in the flexibility that EV fleets can provide to the system. However, participants in long-term tenders bid a single amount of flexibility (in $\mathrm{kW}$ ) for a long period, in advance. Aggregators can thus decide to bid different levels of flexibility according to the risk they are willing to take. Fig. 6 shows the flexibility bid with different levels of certainty by the aggregator (a 0.5 confidence level means the aggregator expects to be able to provide that amount of flexibility $50 \%$ of the time). As higher confidence is required, the aggregator will bid less flexibility, reducing its risk exposure. This can be clearly seen in the amounts bid for the full-day availability window. With a confidence level of 0.5 , EV fleets can bid a certain amount of flexibility with $\mathrm{V} 2 \mathrm{G}$ (over $7 \mathrm{~kW} / \mathrm{EV}$ for the company fleet) as $\mathrm{V} 2 \mathrm{G}$ capacity is available during a significant part of the day, but they cannot provide any amount of flexibility if the reliability requirements are too high (0.99 confidence level). 
Figure 6: Bids [kW/EV] for $0.5,0.9$ and 0.99 confidence levels. Fleet size: 30 EVs

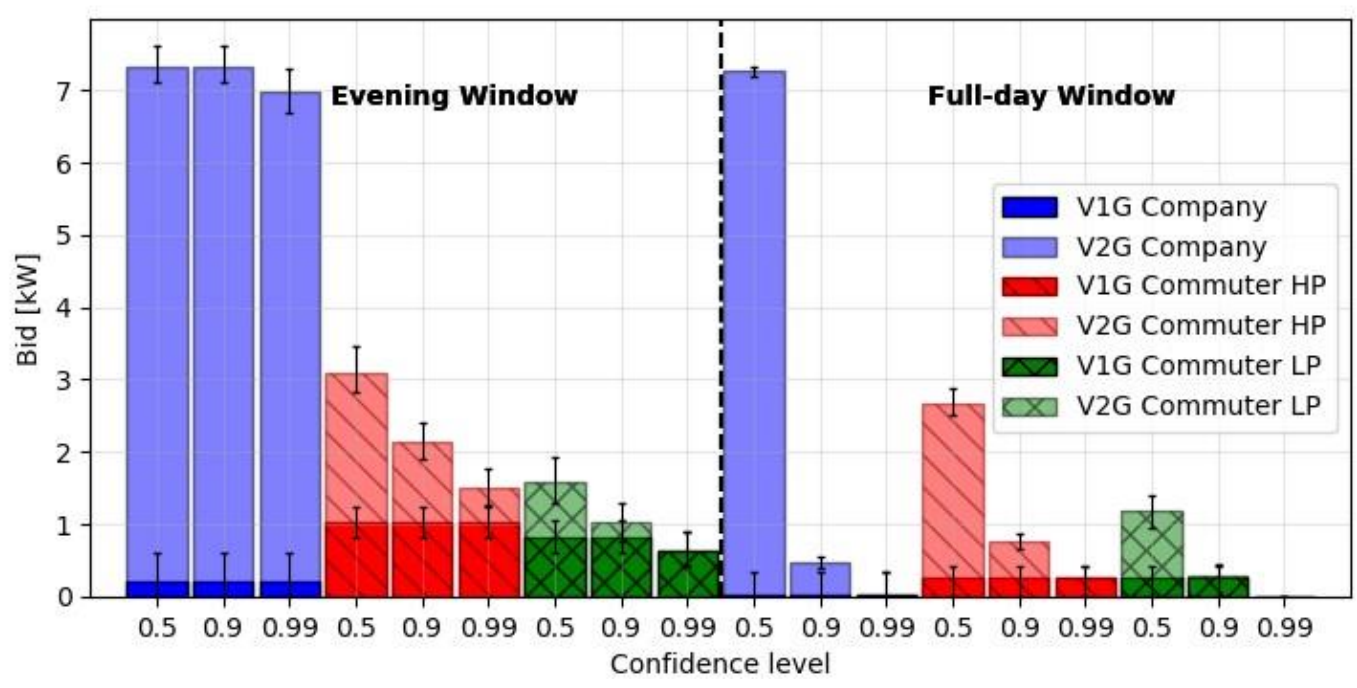

If the fleet is unable to respond to a flexibility activation, it can incur in penalties. UKPN and Enedis have different penalty conditions in their tenders, with UKPN having less strict conditions than Enedis. Fig.7 shows the remuneration for increasing penalty conditions. Under UKPN penalties (upper plot), aggregators face little to no risks, as under-delivery of flexibility does not entail any penalties. As penalty conditions become stricter, aggregators face higher risks ${ }^{10}$. Under Enedis penalties (middle plot), average remuneration is similar for low-confidence bids ( 0.5 confidence) to higher-confidence bids ( 0.9 confidence), but with a higher remuneration spread (whiskers). A risk-averse flexibility operator would logically reduce its bids, bidding only what can be delivered with sufficiently high reliability. Under the extreme scenario, flexibility operators would only bid flexibility with extremely high reliability $(0.99$ confidence), which may preclude access to a significant amount of flexibility resources. Note that under this scenario, even if the flexibility operator is able to cover $89 \%$ of flexibility request, the activation is still not considered valid, and so penalties would apply.

10 Reduced average payments and increasing spread on remuneration. 
Figure 7: Remuneration per EV for three levels of penalty conditions. Fleet size: $30 \mathrm{EVs}$, flexibility price: $€ 50$ per firm $\mathrm{kW}$
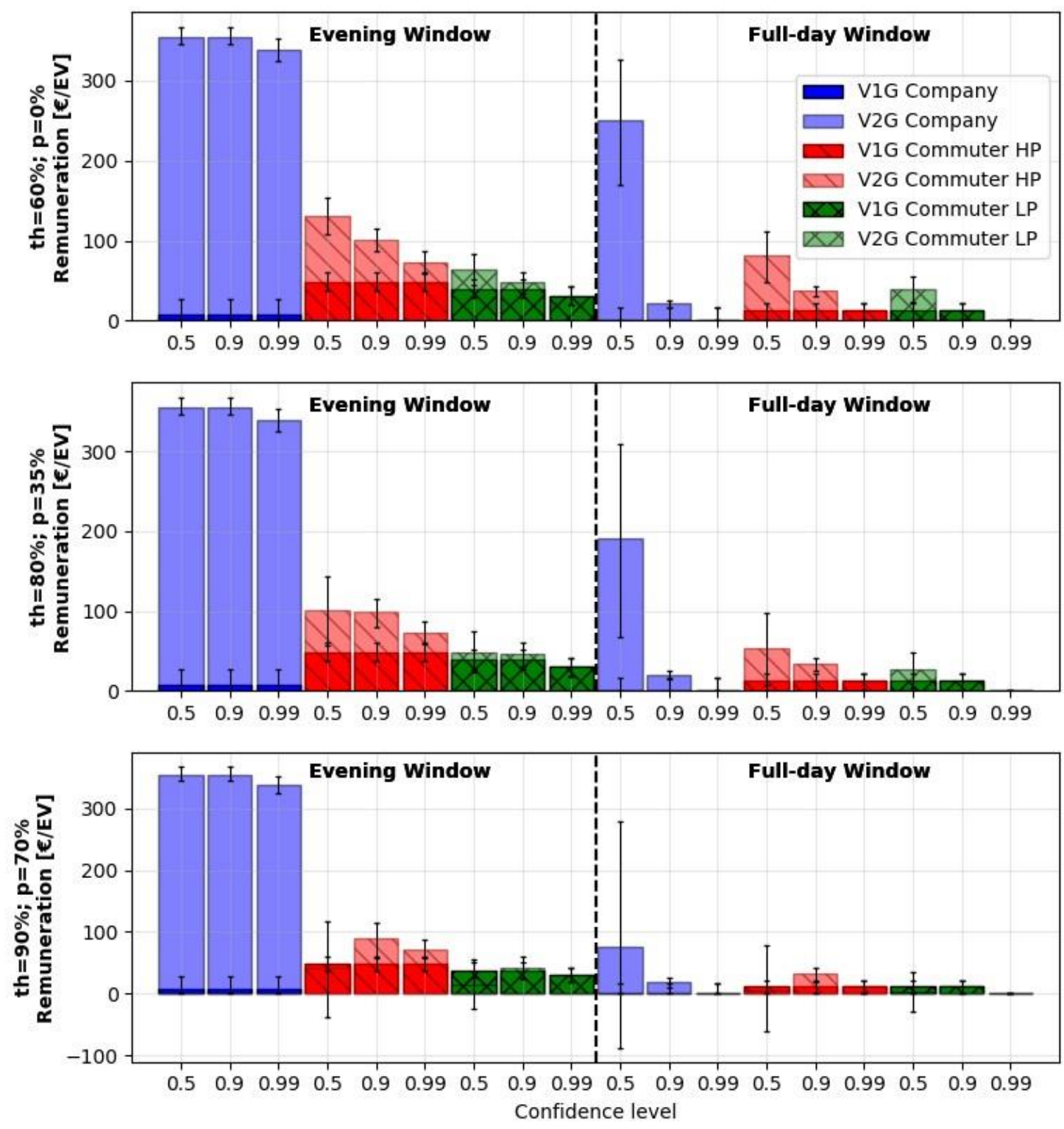

th: Penalty threshold. p: Penalty (in percentage of flexibility price). Whiskers show remuneration spread at $5 \%$ and $95 \%$ confidence.

DSOs will need to balance the need for flexibility reliability with facilitating development of flexibility resources at local level. If penalties are too high, aggregators might not participate in these markets, which would be a serious issue when these markets are only starting. UKPN strategy follows this logic, reducing penalties as a means to reduce barriers to entry and help build liquid markets. Conversely, if penalties are too low, it can put the provision of the flexibility service at risk. We do think that the right level needs to be addressed case-by-case, taking into account local issues, practices and learning by doing for both sides of the relation. 
There are nevertheless alternative solutions to reduce risk exposure for market participants, such as allowing partial participation on the tender (only during certain hours) or defining shorter availability windows that can allow aggregators to better match the availability profiles of their assets to grid requirements. Aggregators can as well, pool different types of resources that can have complementary flexibility profiles to provide flexibility with higher reliability.

\subsection{Remuneration}

Improving EV participation in flexibility tenders hinges on three factors critical: V2G capability, fleet reliability, and a good availability profiles-to-grid requirements match-up. The V2G-capable company fleet obtains over $€ 350 / \mathrm{EV}$ under the evening window that perfectly matches its flexibility profile. However, unreliable commuter fleets (Commuter LP) obtain much lower remuneration, under €50/EV, with most of it coming from V1G. Improving plug-in ratio increases flexibility bids on tenders, thus increasing remuneration from $\mathrm{V} 2 \mathrm{G}$. However, the variability inherent to travel patterns means that the flexibility profile does not match the grid requirements as well as the company fleet (peak availability is reached after 11pm; see Fig. 4).

\subsubsection{Alternative V1G incentives}

The revenue that EVs can expect from V1G only remains limited and comes from charging during (and then displacing the charge from) peak demand hours. Given that time-of-use (ToU, peak/off-peak) tariffs are widely applied in the UK and France, the assumption of uncontrolled charging may not hold, as users already have incentives to charge during off-peak periods. Considering a c€4.5/kWh price difference between peak and off-peak hours ${ }^{11}$ and an EV driving $41 \mathrm{~km} /$ day only during weekdays ${ }^{12}$, charging at off-peak hours would save the end-user $91 € / y^{13}$. These savings could be higher, as electricity tariffs aimed specifically at EV users with higher price differentials (such as super-off-peak tariffs) are commercially available ${ }^{14}$. Electricity tariffs thus provide higher incentives for end-users to charge at offpeak hours on a day-to-day basis than flexibility tenders.

\subsubsection{Sensitivity to flexibility price}

The value of flexibility can vary greatly according to tender conditions, as shown in Fig. 1. In the UKPN $\mathrm{HV}$ tenders, flexibility is valued against the annualized costs of alternative infrastructure reinforcements. The equivalent price for flexibility (in $€$ or $€ / M W / h$ ) can be derived from the expected magnitude of the constraints (in MW) and duration of the availability service (in hours). In the UKPN case, flexibility value ranges from 3.25 to over $4500 \mathrm{f} / \mathrm{MW} / \mathrm{h}$. Andrianesis et al. (2019) proposed a similar methodology to value flexibility for reinforcement deferral and applied it to a representative US MV grid, obtaining values ranging from $\$ 200 / \mathrm{MW} / \mathrm{h}$ to over $\$ 4000 / \mathrm{MW} / \mathrm{h}$ during constrained periods that occur only a few hours a year. With these approaches, the value of flexibility decreases with increasing grid constraint (in time and power magnitude) as it becomes more cost-efficient to invest in reinforcement. This means that distribution grid services become less attractive compared to other flexibility services such as frequency regulation (i.e. the opportunity cost for a flexibility operator participating in local tenders grows with respect to alternative services).

11 Tarif bleu, a regulated tariff in France provided by EDF, 2020 (EDF (2020))

12 Average daily distance in France (Borne (2019)). Similar values are found in other European countries

13 Considering EV efficiency: $0.18 \mathrm{kWh} / \mathrm{km}$, EVSE efficiency: 0.95

14 For example, in the UK, Octopus Energy offers a super-off-peak tariff with 9.7 p/kWh price difference (Octopus Energy (2020)), and in France, Engie offers an EV tariff with $7.4 \mathrm{c} € / \mathrm{kWh}$ price difference (ENGIE (2020)) 
Given the large flexibility value spread, we carried out a sensitivity analysis of total remuneration per EV for the studied tenders (Table 6). High and low values are representative of UKPN's high and low value areas. It should be noted as well that Enedis-led studies put flexibility for investment deferral in a range of 0-24 $€ / \mathrm{kW}$ for the value of flexibility for investment deferral Enedis (2019), which lies on the lower end of UKPN's tender processes.

Total remuneration can reach over $€ 1400 / \mathrm{EV} / \mathrm{y}$ in favorable tender conditions (company fleet participating in evening window with high flexibility value, ). This is in the same order of magnitude than year-round PFC services revenues. Calearo and Marinelli (2020) ) showed average revenues of $€ 1322 € / E V / y$ for providing frequency regulation services 13 hours per day, every day, in Denmark's DK2 area ${ }^{15}$, and Borne et al. (2018b) showed best-case revenues of $€ 1000 / E V / y$ in the FCR cooperation area $^{16}$. PFC revenues can be complementary to distribution investment deferral services, as they are needed only a few hours a year.

Table 6: Average remuneration per EV [ $€ / \mathbf{y}]$. Sensitivity analysis on price per firm $\mathbf{k W}$. Highlighted cells provide the highest remuneration for each type of fleet

\begin{tabular}{lrcccccc}
\hline & & \multicolumn{2}{c}{ Company } & \multicolumn{2}{c}{ Commuter HP } & \multicolumn{2}{c}{ Commuter LP } \\
\multicolumn{2}{l}{ Price $[€ / \mathbf{k W}]$} & Evening & Full-day & Evening & Full-day & Evening & Full-day \\
\hline \multirow{2}{*}{ V1G } & 12.5 & 2.5 & 3.1 & 12.0 & 4.3 & 9.5 & 4.0 \\
& $\mathbf{5 0}$ & $\mathbf{1 0 . 2}$ & $\mathbf{1 2 . 7}$ & $\mathbf{4 8 . 2}$ & $\mathbf{1 7 . 3}$ & $\mathbf{3 8 . 1}$ & $\mathbf{1 6 . 0}$ \\
& 200 & 40.8 & 50.8 & 192.8 & 69.2 & 152.4 & 64.0 \\
\hline \multirow{2}{*}{ V2G } & 12.5 & 88.9 & 5.2 & 25.6 & 9.1 & 12.1 & 3.2 \\
& $\mathbf{5 0}$ & $\mathbf{3 5 5 . 8}$ & $\mathbf{2 1 . 1}$ & $\mathbf{1 0 2 . 5}$ & $\mathbf{3 6 . 5}$ & $\mathbf{4 8 . 4}$ & $\mathbf{1 3 . 1}$ \\
& 200 & 1423.2 & 84.4 & 410.0 & 146.0 & 193.6 & 52.4 \\
\hline
\end{tabular}

\subsubsection{Impact of fleet sizes}

Fleet size can have significant impact on the amount of flexibility that can be delivered with a given confidence level. Fig. 8 shows the remuneration obtained for different fleet sizes. As fleet sizes grow, the aggregated charging and travel patterns become more reliable, allowing aggregators to bid higher amounts of flexibility on tenders (in $\mathrm{kW} / \mathrm{EV}$ ) and reducing revenue variability. For the evening window where EV patterns are more reliable (above $30 \mathrm{EVs}$ available for the company fleet and $50 \mathrm{EVs}$ for commuter fleets), these revenues show little change. For the full-day window pooling above $100 \mathrm{EVs}$ per fleet, the revenues remain relatively stable. Note that attainable revenues are lower for full-day windows than for the evening window, as EVs cannot reliably provide flexibility during the whole day.

15 PFC prices in DK2 area range between 12-60 €/MW/h, much higher than in the UK or Continental Europe. workplace (with $22 \mathrm{~kW}$ charger). 


\section{Figure 8: Remuneration per EV for different fleet sizes, evening window (left) and full-day window (right)}
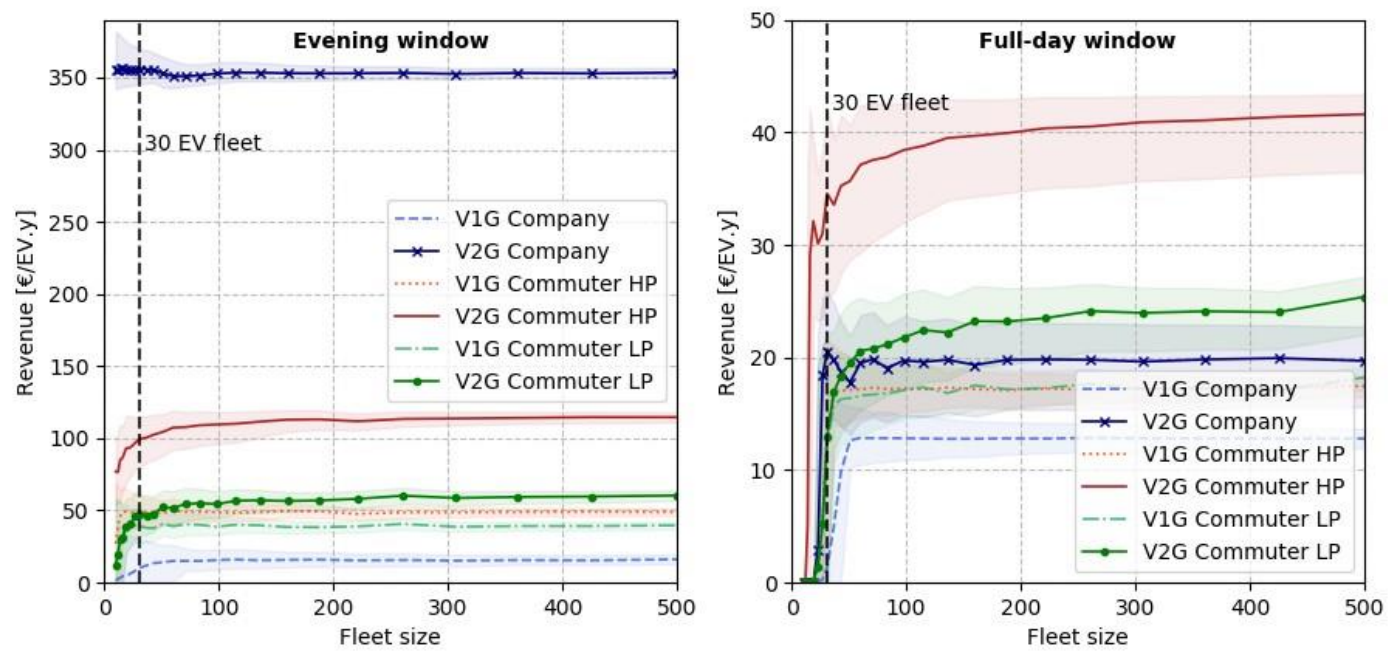

Note different scales on remuneration. Flexibility price: $€ 50 / \mathrm{kW}$; Penalty threshold: 80 ; Penalty value: $35 \%$

\section{Conclusions and Policy Implications}

DSOs are starting to implement market-based mechanisms to use the flexibility offered by DERs. Recent implementations in Europe reflect a wide array of solutions tested, most of which are respond to the technical problems encountered in local distribution grids. In zones presenting mainly RES generationdriven congestion, short-term market platforms have been implemented to reduce uneconomic curtailment, whereas in zones expecting load growth, medium- to long-term tenders have been implemented to defer or avoid costly infrastructure reinforcements.

Long-term tenders provide valuable certainty for DSOs and flexibility operators and encourage the development of flexibility trading at local level, and can thus be the first step towards building liquid short-term flexibility markets. In this context, the London-area DSO

UKPN has been procuring flexibility through tenders since 2018, and the recent April 2020 UKPN tender demonstrates the high potential value of flexibility for investment deferral with a significant share of flexible contracts awarded to EV aggregators. Here we proposed a modular framework to identify the main barriers to participation of DERs on local flexibility markets and applied it to five emerging platforms. The main barriers are on high minimum bid requirements and on connection and metering equipment, as most of these solutions are still aimed at medium-size and large customers. We presented a model to quantify the participation of EVs in local flexibility tenders. The two-stage model emulates the (ex-ante) tender bidding and activation-settlement processes. We then applied the model to three types of EV fleets using real-life data from European demonstrator projects. Our case study considered EVs, but our methodology can be applied to other types of DER, such as residential demand response.

Our results identify three main factors affecting flexibility provision and remuneration by EV fleets: bidirectional capability ( $\mathrm{V} 2 \mathrm{G})$, reliability of the fleet, and the availability profiles-tender requirements match-up. Minimum service time has little impact, as EV fleets can provide 2-hour service with almost the same reliability as 30-minute service. Aggregators looking to make use of EV flexibility should focus on fleets that have consistent and reliable usage profiles, and they should probably also incentivize end-user plug-ins. 
V2G-capable EV fleets in high-value areas with a good match of availability profile to tender requirements can obtain revenues of over $€ 1400 / \mathrm{EV}$ per year providing services for only a few hours or months per year. This revenue stream can be complementary to other flexibility services such as frequency regulation. However, these revenues are dependent on grid context: not all grids are constrained, constraints differ in each case (in time, magnitude, duration and frequency), and as constraints grow in time and magnitude, the value of flexibility diminishes as it becomes more costeffective to use alternative reinforcement solutions.

Future research could usefully explore flexibility provision by other types of EV fleets such as public charging infrastructure or office parking lots, as these types of fleets have different occupancy patterns that can meet other types of grid requirements. Shorter charging sessions can also put additional constraints on flexibility provision. Second, research would benefit from a methodology to define optimal bidding for EV fleets participating in local flexibility tenders. Inspiration can be found in the abundant research on optimal bidding for electricity market participants.

We conclude with policy recommendations on local flexibility markets. First, local flexibility markets are not built overnight; they require strong collaboration between regulators, DSOs and flexibility operators. Regulatory frameworks are needed to push DSOs to procure flexibility when costeffective instead of investing in infrastructure, and DSOs should take a proactive approach to building these markets, listening to stakeholders and sharing data. In this regard, UKPN has led an open and transparent process, identifying potential tender zones months or years in advance and publishing potential revenues for each tendered zone, which helps flexibility operators identify the value of flexibility.

Second, tender conditions should be adapted to facilitate the participation of DER aggregators. On the administrative front, easing connection requirements, using existing metering equipment (such as smart meters) and having broad support from local-country DSOs (such as with the PicloFlex platform or the Enedis case) can help reduce barriers to entry for new aggregators. On the product definition front, a clear barrier is the minimum bid, which should be as low as possible (ideally under $50 \mathrm{~kW}$ ) to allow the participation of several aggregators and foster competition. Furthermore, setting short availability windows can help engage participation of assets with variable availability patterns (such as EVs and demand response) to match grid requirements. Finally, on the payments front, long-term contracts should consider both activation and availability payments to secure available flexibility, along with penalties applicable in the event of failure to deliver. However, penalties should not be excessive as to discourage the participation of flexibility aggregators, and should consider local issues, practices and learning by doing for both sides of the relation. 


\section{References}

Andersen PB, Sousa T, Thingvad A, Berthou LS, Kulahci M. Added Value of Individual Flexibility Profiles of Electric Vehicle Users for Ancillary Services. In: 2018 IEEE International Conference on Communications, Control, and Computing Technologies for Smart Grids, SmartGridComm 2018; 2018. doi:10.1109/SmartGridComm.2018.8587585.

Andrianesis P, Caramanis M, Masiello R, Tabors R, Bahramirad S. Locational Marginal Value of Distributed Energy Resources as Non-Wires Alternatives. IEEE Transactions on Smart Grid 2019;1doi:10.1109/tsg.2019.2921205. arXiv:1811.09006.

Banol Arias N, Hashemi S, Andersen PB, Traholt C, Romero R. V2G enabled EVs providing frequency containment reserves: Field results. In: Proceedings of the IEEE International Conference on Industrial Technology. 2018-2; 2018. p. 1814-9. doi:10.1109/ ICIT.2018.8352459.

Berthou L. Flexibility Profiles for EV Users. Ph.D. thesis; Technical University of Denmark; 2018.

Borne O. Vehicle-To-Grid and Flexibility for Electricity Systems: from Technical Solutions to Design of Business Models. Ph.D. thesis; Université Paris-Saclay; 2019.

Borne O, Korte K, Perez Y, Petit M, Purkus A. Barriers to entry in frequency-regulation services markets: Review of the status quo and options for improvements. Renewable and Sustainable Energy Reviews 2018a; 81:605-14. doi:10.1016/J.RSER.2017.08.052.

Borne O, Perez Y, Petit M. Market integration or bids granularity to enhance flexibility provision by batteries of electric vehicles. Energy Policy 2018b; 119:140-8. doi:10. 1016/j.enpol.2018.04.019.

Calearo L, Marinelli M.Profitability of Frequency Regulation by Electric Vehicles in Denmark and Japan Considering Battery Degradation Costs. World Electric Vehicle Journal 2020;11(3):48. doi:10.3390/ wevj11030048.

Cedec , Edso , Entso-e , Eurelectric , Geode . TSO-DSO Report: An Integrated Approach to Active System Management. Technical Report; 2019.

Codani P. Integration des véhicules électriques dans les réseaux électriques : Modèles d'affaire et contraintes techniques pour constructeurs automobiles. Ph.D. thesis; Université Paris-Saclay; 2016. URL: http://www.theses.fr/2016SACLC073.

Codani P, Perez Y, Petit M. Financial shortfall for electric vehicles: Economic impacts of Transmission System Operators market designs. Energy 2016;113:422-31. doi:10.1016/ j.energy.2016.07.070.

Commission de Régulation de l'Energie. Les réseaux électriques au service des véhicules électriques. Technical Report; 2018.

Council of European Energy Regulators. Flexibility Use at Distribution Level A CEER Conclusions Paper. Technical Report; 2018.

EDF. Tarif Bleu. 2020. URL: https://particulier.edf.fr/content/dam/2-Actifs/ Documents/Offres/Grille_prix_Tarif_Bleu.pdf.

EDSO, CEDEC , Eurelectric, GEODE . Flexibility in the Energy Transition: A Toolbox for Electricity DSOs. Technical Report; 2018.

Enedis . Flexibilities to enhance the Energy Transition and the performance of the Distribution Network. Technical Report; 2019.

Enedis. Feuille de route pour la transformation des méthodes de dimensionnement des réseaux et l'intégration des flexibités. Technical Report; 2020a.

Enedis . Modèles de contrats Présentation des principaux éléments contractuels. Technical Report; $2020 \mathrm{~b}$. 
Engelbrecht D. Schweer, A., Gehrcke, R., Lauen, E., Deuchert, B., Wilczek, J., Schuster, H., Büchner, J. Demonstration of a Market-based Congestion Management using a Flexibility Market in Distribution Networks. In: Internationaler ETG-Kongress. Esslingen am Neckar; 2019. p. 306-11.

ENGIE. Elec Car : $-50 \%$ sur l'électricité la nuit pour recharger votre voiture. 2020. URL: https://particuliers.engie.fr/electricite/voitures-electriques/ contrat-elec-car.html.

EPEX Spot. enera project: EWE and EPEX SPOT to create local market platform to relieve grid congestions. 2018.

Everoze, EVConsult. V2G Global Roadtrip: Around the World in 50 Projects. Technical Report October; 2018.

Fonteijn R, Nguyen P, Morren J, Slootweg J. Demonstrating a generic four-step approach for applying flexibility for congestion management in daily operation. Sustainable Energy, Grids and Networks 2020;:100378 doi:10.1016/j.segan.2020.100378.

Gonzalez Venegas F, Petit M, Perez Y. Can DERs fully participate in emerging local flexibility tenders? In: International Conference on the European Energy Market, EEM. Ljubljana; 2019; 2019a. doi:10.1109/EEM.2019.8916343.

Gonzalez Venegas F, Petit M, Perez Y. Electric Vehicles as Flexibility Providers for Distribution Systems. A Techno-Economic review. In: Proc. of the 25th International Conference on Electricity Distribution (CIRED 2019). Madrid; 2019b. p. 3-6.

Gonzalez Venegas F, Petit M, Perez Y. Active integration of electric vehicles into distribution grids: barriers and frameworks for flexibility services; Under review. 2020a.

Gonzalez Venegas F, Petit M, Perez Y. Quantifying the participation of EV fleets in local flexibility tenders. In: Proc. of CIRED 2020 Berlin Workshop. Berlin; 2020b. .

GOPACS.eu. Public Announcements. 2020. URL: https://en.gopacs.eu/publicannouncements/.

International Energy Agency. Global EV Outlook 2020. Technical Report; 2020.

Jin X, Wu Q, Jia H. Local flexibility markets: Literature review on concepts, models and clearing methods. 2020. doi:10.1016/j.apenergy.2019.114387.

Knezovic K, Marinelli M, Codani P, Perez Y. Distribution grid services and flexibility provision by electric vehicles: A review of options. In: Proceedings of the Universities Power Engineering Conference. IEEE Computer Society; 2015-11; 2015. doi:10.1109/UPEC.2015.7339931.

Knezovic K, Marinelli M, Zecchino A, Andersen PB, Traeholt C. Supporting involvement of electric vehicles in distribution grids: Lowering the barriers for a proactive integration. Energy 2017;134:458-68. doi:10.1016/j.energy.2017.06.075.

National Grid ESO. Firm frequency response (FFR). 2020. URL: https://www.nationalgrideso.com/industry-information/balancing-services/frequency-responseservices/firm-frequency-response-ffr? market-information.

Nuvve Corp. PSA GridMotion Project - NUVVE Corp. 2017. URL:

http://nuvve.com/portfolio/gridmotion-project-reducing-electric-vehicle-usage-cost-thanks-tosmart-charging-proc Octopus Energy .

Octopus Go. 2020. URL: https://octopus.energy/go/.

Olivella-Rosell P, Lloret-Gallego P, Munné-Collado I, Villafafila-Robles R, Sumper A, Ottessen SØ, Rajasekharan J, Bremdal BA. Local flexibility market design for aggregators providing multiple flexibility services at distribution network level. Energies 2018;11(4). doi:10.3390/en11040822. 
REV Connect . Non-wires alternatives. 2020. URL:

https://nyrevconnect.com/non-wiresalternatives/.

Rossetto N. Measuring the Intangible: An Overview of the Methodologies for Calculating Customer Baseline Load in PJM. FSR Policy Briefs 2018; URL: https://www.ferc.gov/ industries/electric/indus-act/demand-response/dem-res-adv-metering.asp.

RTE . Règles relatives a' la Programmation, au Mécanisme d'Ajustement et au Recouvrement des charges d'ajustement. Technical Report; 2020.

RTE Portail Service . Téléchargez les données publiées par RTE. 2020. URL: https: //www.services-rte.com/fr/telechargez-les-donnees-publiees-par-rte.html? category=market\&type=balancing_capacity\&subType=procured_reserves.

Schittekatte T, Meeus L. Flexibility markets: Q\&A with project pioneers. Utilities Policy 2020;63:101017. doi:10.1016/j.jup.2020.101017.

Thompson AW, Perez Y. Vehicle-to-Everything (V2X) energy services, value streams, and regulatory policy implications. Energy Policy 2019;137:111136. doi:10.1016/j.enpol. 2019.111136.

UK Power Networks. Future Smart: Flexibility Roadmap. Technical Report; 2018.

UK Power Networks. Post Tender Report. Technical Report; 2020. URL: https:// smartgrid.ukpowernetworks.co.uk/flexibility-hub/.

Wang D, Coignard J, Zeng T, Zhang C, Saxena S. Quantifying electric vehicle battery degradation from driving vs. vehicle-to-grid services. Journal of Power Sources 2016;332:193-203. doi:10.1016/j.jpowsour.2016.09.116.

Western Power Distribution . Electric Nation Data. URL: https://www.westernpower. co.uk/electricnation-data.

Western Power Distribution . Electric Nation Customer Trial Final Report. Technical Report; 2019.

Williamson OE. The Economic Institutions of Capitalism: Firms, Markets, Relational Contracting. Free Press, 1985.

Williamson OE. The Mechanisms of Governance. Oxford University Press, 1996. 


\section{Author contacts:}

\section{Felipe Gonzalez Venegas (corresponding author)}

Université Paris-Saclay, CentraleSupélec, CNRS

Laboratoire de Génie Electrique et Electronique de Paris

91192, Gif-sur-Yvette

France

and

Innovation \& Advanced Technologies Research Division, Groupe PSA

78955, Carrières-sous-Poissy

France

Email: felipe.gonzalezvenegas@centralesupelec.fr

\section{Marc Petit}

Université Paris-Saclay, CentraleSupélec, CNRS

Laboratoire de Génie Electrique et Electronique de Paris

91192, Gif-sur-Yvette

France

\section{Yannick Perez}

Université Paris-Saclay, CentraleSupélec, Laboratoire de Génie Industriel 91190 Gif-sur-Yvette

France 
With the support of the The European Commission supports the EUI through the European Union budget. This Erasmus+ Programme publication reflects the views only of the author(s), and the Commission cannot be held of the European Union responsible for any use which may be made of the information contained therein. 